\title{
Aneurysm Morphology and Prediction of Rupture: An International Study of Unruptured Intracranial Aneurysms Analysis
}

\section{J. Mocco, MD, MS* \\ Robert D Brown, Jr, MD, MPH James C. Torner, PhD ${ }^{\S}$ \\ Ana W. Capuano, PhD" \\ Kyle M. Fargen, MD, MPH" \\ Madhavan L. Raghavan, PhD\# \\ David G. Piepgras, MD** \\ Irene Meissner, MD $^{\ddagger}$ \\ John Huston III, MD \\ on behalf of the International \\ Study of Unruptured \\ Intracranial Aneurysms \\ Investigators}

*Department of Neurosurgery, Mount Sinai Medical Center, New York, New York; ${ }^{\ddagger}$ Department of Neurology, Mayo Clinic, Rochester, Minnesota; ${ }^{\S}$ Department of Epidemiology, University of lowa, lowa City, lowa; "Rush Alzheimer's Disease Center, Rush University Medical Center, Chicago, IL; "Department of Neurosurgery, Wake Forest University, Winston-Salem, North Carolina; "Department of Biomedical Engineering, University of lowa, lowa City, lowa; **Department of Neurosurgery, Mayo Clinic, Rochester, Minnesota; \#‡Department of Radiology, Mayo Clinic, Rochester, Minnesota

\section{Correspondence:}

Kyle M. Fargen, MD, MPH, Department of Neurosurgery, Wake Forest University, 1 Medical Center Blvd,

Winston-Salem, NC 27157.

E-mail:kfargen@wakehealth.edu

Received, July 31, 2016.

Accepted, May 24, 2017.

Published Online, June 10, 2017.

Copyright (c) 2017 by the

Congress of Neurological Surgeons

BACKGROUND: There are conflicting data between natural history studies suggesting a very low risk of rupture for small, unruptured intracranial aneurysms and retrospective studies that have identified a much higher frequency of small, ruptured aneurysms than expected.

OBJECTIVE: To use the prospective International Study of Unruptured Intracranial Aneurysms cohort to identify morphological characteristics predictive of unruptured intracranial aneurysm rupture.

METHODS: A case-control design was used to analyze morphological characteristics associated with aneurysm rupture in the International Study of Unruptured Intracranial Aneurysms database. Fifty-seven patients with ruptured aneurysms during follow-up were matched (by size and location) with 198 patients with unruptured intracranial aneurysms without rupture during follow-up. Twelve morphological metrics were measured from cerebral angiograms in a blinded fashion.

RESULTS: Perpendicular height $(P=.008)$ and size ratio (ratio of maximum diameter to the parent vessel diameter; $P=.01$ ) were predictors of aneurysm rupture on univariate analysis. Aspect ratio, daughter sacs, multiple lobes, aneurysm angle, neck diameter, parent vessel diameter, and calculated aneurysm volume were not statistically significant predictors of rupture. On multivariate analysis, perpendicular height was the only significant predictor of rupture (Chi-square 7.1, $P$-value .008).

CONCLUSION: This study underscores the importance of other morphological factors, such as perpendicular height and size ratio, that may influence unruptured intracranial aneurysm rupture risk in addition to greatest diameter and anterior vs posterior location.

KEY WORDS: Aneurysm, Cerebrovascular circulation, Subarachnoid hemorrhage, Morphology, Cerebral aneurysm, Hemorrhagic stroke, Natural history, Unruptured

Neurosurgery 82:491-496, 2018

DOI:10.1093/neuros/nyx226

www.neurosurgery-online.com

$\mathbf{T}$ here is an ongoing debate regarding the rupture risk of unruptured intracranial aneurysms (UIA). To date, the most robust data describing the natural history of UIA derive from several longitudinal studies. ${ }^{1-4}$ These and other studies have consistently suggested that larger aneurysm size is an important risk factor for rupture. ${ }^{1-3,5}$ Furthermore, aneurysm location has also been noted to be a potential predictor of aneurysm rupture. ${ }^{1,2,4-6}$ Given

ABBREVIATIONS: ISUIA, International Study of Unruptured Intracranial Aneurysms; SAH, subarachnoid hemorrhage; SR, size ratio; UIA, unruptured intracranial aneurysms that the treatment of UIA by surgical clipping or endovascular coiling is associated with the potential for morbidity and mortality, ${ }^{2}$ defining readily identifiable factors that impact on the risk profile of UIA is important for counseling patients regarding treatment options.

Complicating our understanding of UIA risk are the conflicting data between natural history studies, suggesting a very low risk of rupture for small $(<7 \mathrm{~mm})$ UIA and retrospective studies that have identified a much higher frequency of small, ruptured aneurysms than expected. For instance, the International Study of Unruptured Intracranial Aneurysms (ISUIA) observed a $0.05 \%$ rupture risk of small $(<7 \mathrm{~mm})$ anterior circulation aneurysms over $5 \mathrm{yr}^{1,2}$ However, 


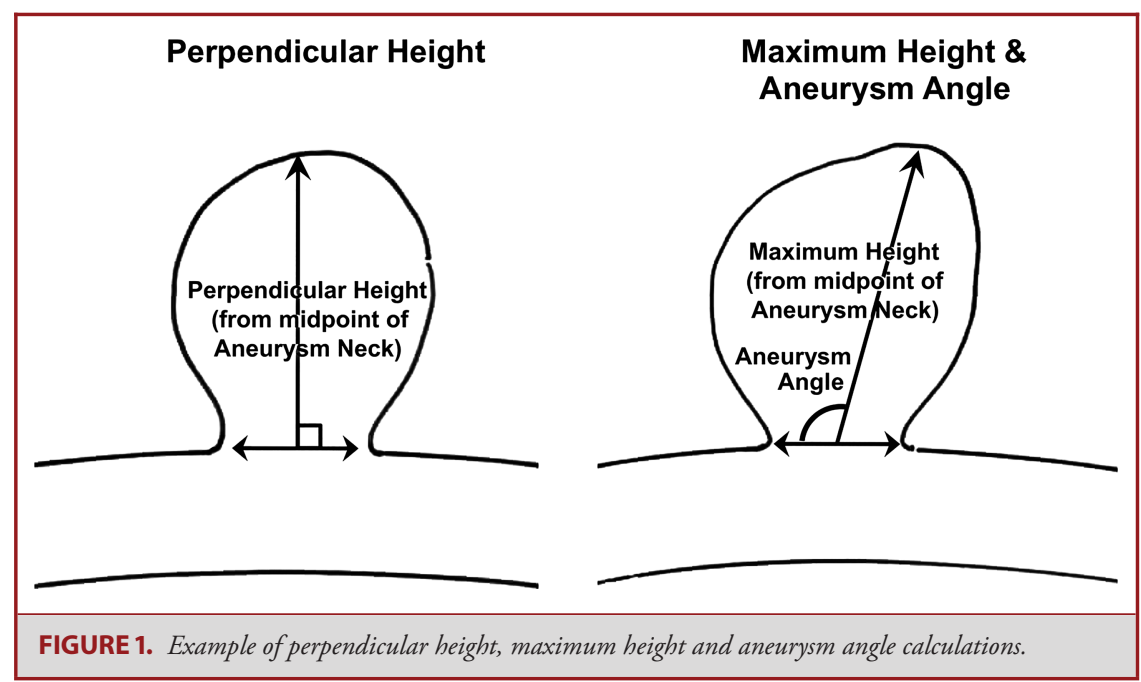

in a study of almost 1000 patients presenting with ruptured aneurysms, Weir and colleagues 5 showed that $70 \%$ of ruptured aneurysms were less than $10 \mathrm{~mm}$ in size. Furthermore, Carter et $\mathrm{al}^{6}$ have suggested that the sizes of ruptured aneurysms differ based on location and are smaller on more distal arteries than those arising from a more proximal location. The difficulty in reconciling the major natural history studies with observational studies of patients with ruptured aneurysms suggests that other morphological characteristics, besides just maximum diameter or anterior vs posterior circulation, may be important. Other aneurysm morphology characteristics have been identified as potential predictors of UIA rupture, including size ratio (SR). ${ }^{7,8}$

The purpose of the present study is to use the prospective ISUIA cohort to identify potential morphological characteristics predictive of aneurysm rupture to gain further insight into the risk profile of UIA.

\section{METHODS}

\section{Database}

ISUIA is a prospective epidemiological cohort study of patients with UIA, with 61 participating centers in North America and Europe. Patients were prospectively consented and enrolled from 1991 to1998 (phase I and II). All patients had 2-dimensional angiographic images defining the presence of a UIA available for central review at the ISUIA Coordinating Center at Mayo Clinic, Rochester, Minnesota. Phase III involved annual follow-up of the prospective cohort from 2004 to 2007, and included 1917 patients surgically treated with clipping, 451 treated with endovascular techniques, and 1691 that were prospectively observed. Detailed methodology, including aneurysm measurement technique, and results of the initial phases may be found elsewhere. $^{1,2}$

\section{Present Study}

Institutional review board approval was obtained. A nested casecontrol design was used to analyze morphological characteristics associated with aneurysm rupture in the ISUIA database. From 1691 patients in the prospective cohort, 57 patients with ruptured aneurysms of all sizes during follow-up were then matched with 198 patients with UIA without rupture during follow-up by both maximum aneurysm diameter $( \pm 2 \mathrm{~mm})$ and parent artery (location). This study was designed to examine morphological and shape parameters. Of note, nonmorphological characteristics such as: age, gender, smoking history, subarachnoid hemorrhage (SAH) history, cerebrovascular disease, hypertension, transient ischemic attack history, atrial fibrillation, congestive heart failure history, and use of aspirin were also analyzed to ensure no potentially significant confounders were missed. All cerebral angiograms obtained at initial presentation were reviewed and measured in a blinded fashion. Two independent investigators (primary and last author) reviewed all angiograms separately and any discrepancies were resolved by combined review and consensus. Aneurysm morphological characteristics recorded included: (1) maximum diameter; (2) aneurysm angle; (3) parent vessel location; (4) neck diameter; (5) presence of daughter sac (defined as being $<50 \%$ of the parent aneurysm size); (6) presence of multiple lobes (defined as being $>50 \%$ of the parent aneurysm size); (7) aspect ratio (ratio of aneurysm maximum height to neck diameter); (8) maximum height (maximum measurement from the center of the aneurysm neck to the aneurysm dome; Figure 1); (9) SR (ratio of maximum diameter in any direction to the parent vessel diameter[s], wherein both branching vessels and inflow vessel sizes were averaged for bifurcating aneurysms; Figure 2); (10) parent vessel diameter; (11) perpendicular height (the measurement of the aneurysm height at a perpendicular to the center of the aneurysm neck to the aneurysm dome; Figure 1); (12) aneurysm volume, calculated by $(4 / 3) \pi\left(\right.$ radius $\left.^{\mathrm{a}}\right)\left(\right.$ radius $\left.^{\mathrm{b}}\right)\left(\right.$ radius $\left.^{\mathrm{c}}\right)$, where $\mathrm{a}, \mathrm{b}$, and $\mathrm{c}$ are the orthogonal measurements.

\section{Statistics}

Univariate and multivariate conditional logistic regression were performed to identify factors associated with aneurysm rupture. Variables were analyzed in a systematic fashion, with binomial variables being identified as present/absent, while continuous variables were subgrouped with a priori designations to create a reasonable distribution of data points across each subgroup. Variables chosen for inclusion in the multivariable analysis were identified, secondary to being statistically 


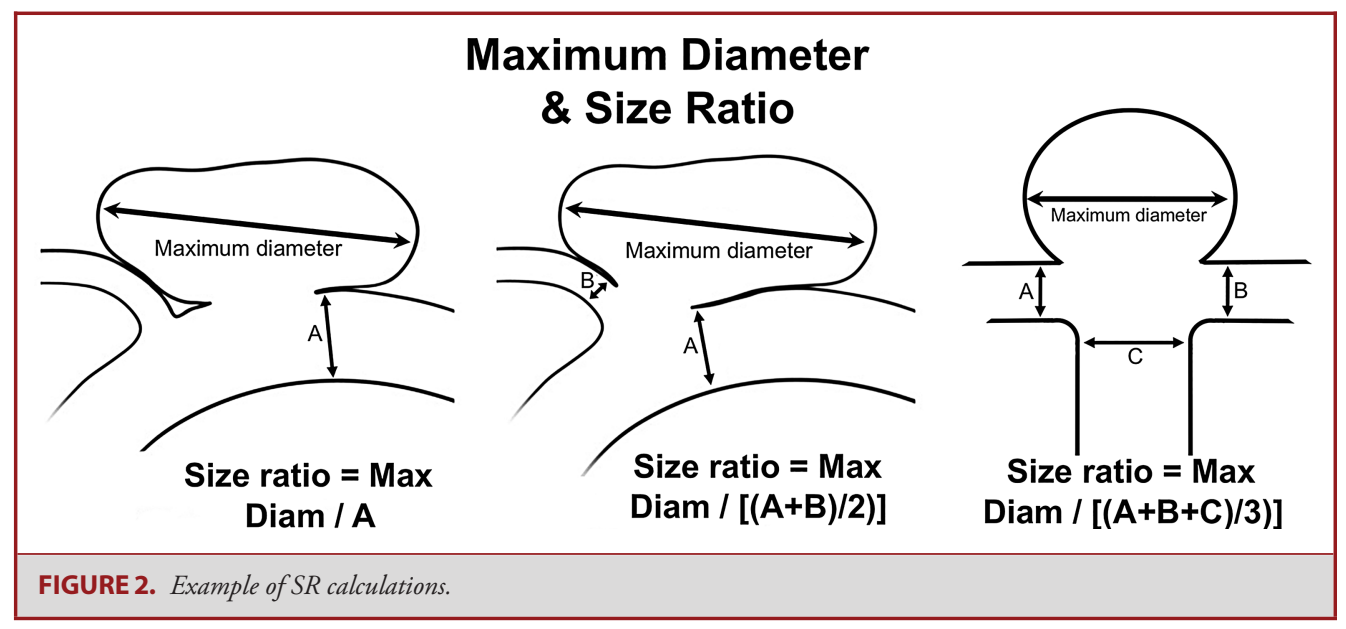

significant in univariable analysis. A $P$-value of .05 was chosen for statistical significance.

\section{RESULTS}

\section{Descriptive Data}

A total of 57 previously UIA that ruptured during follow-up were matched by size and location to 198 aneurysms that did not rupture during follow-up. The distribution of aneurysm sizes and locations for cases and controls are shown in Table 1.

\section{Main Results}

Morphological findings for cases and controls and results of univariate analyses are summarized in Table 2. Of note, nonmorphological characteristics such as: age, gender, region, smoking history, SAH history, cerebrovascular disease, hypertension, transient ischemic history, atrial fibrillation, congestive heart failure history, and use of aspirin were nonsignificant. Only perpendicular height and SR were predictors of aneurysm rupture on univariate analysis. Other factors including aspect ratio, presence of daughter sac, presence of multiple lobes, aneurysm angle, neck diameter, parent vessel diameter, and calculated aneurysm volume were not statistically significant predictors of rupture. On multivariate conditional logistic regression, adjusting for family history and smoking, perpendicular height was the only significant predictor of rupture (Chi-square 4.01, $P$-value .0451; all others $P$-value greater than .05).

\section{DISCUSSION}

\section{Key Results}

Studies on UIA have consistently suggested that larger aneurysm size ${ }^{1-3,5}$ and aneurysm location ${ }^{1,2,4-6}$ are important predictors of future aneurysm rupture. When both aneurysm size and aneurysm parent vessel location were controlled for in this

\begin{tabular}{|c|c|c|}
\hline & $\begin{array}{c}\text { Controls } \\
n=198\end{array}$ & $\begin{array}{l}\text { Cases } \\
n=57\end{array}$ \\
\hline \multicolumn{3}{|l|}{ Age } \\
\hline$<50 \mathrm{yr}$ & 62 & 12 \\
\hline $50-69 \mathrm{yr}$ & 101 & 30 \\
\hline $70+y r$ & 35 & 15 \\
\hline \multicolumn{3}{|l|}{ Gender } \\
\hline Male & 45 & 16 \\
\hline Female & 153 & 41 \\
\hline \multicolumn{3}{|c|}{ Size (largest diameter, any direction) } \\
\hline$<3 \mathrm{~mm}$ & 8 & 1 \\
\hline 3-5 mm & 24 & 9 \\
\hline $6-8 \mathrm{~mm}$ & 25 & 7 \\
\hline 9-11 mm & 39 & 7 \\
\hline $12-14 \mathrm{~mm}$ & 37 & 12 \\
\hline $15-25 \mathrm{~mm}$ & 40 & 9 \\
\hline$>25 \mathrm{~mm}$ & 25 & 12 \\
\hline \multicolumn{3}{|l|}{ Location } \\
\hline Internal carotid & 58 & 7 \\
\hline Anterior cerebral/ACOM & 8 & 7 \\
\hline Middle cerebral & 29 & 9 \\
\hline Basilar trunk & 2 & 2 \\
\hline Posterior communicating & 49 & 18 \\
\hline Posterior cerebral & 5 & 1 \\
\hline Basilar apex & 31 & 11 \\
\hline Other posterior circulation & 16 & 2 \\
\hline
\end{tabular}

ACOM, anterior communicating artery

analysis of the ISUIA cohort using a case-control design with matching, perpendicular height was identified as an additional factor that predicted aneurysm rupture on multivariate analysis. The overall effect of this factor on rupture risk when including 


\begin{tabular}{|c|c|c|c|}
\hline & $\begin{array}{c}\text { Controls } \\
n=198\end{array}$ & $\begin{array}{l}\text { Cases } \\
\mathrm{n}=57\end{array}$ & $P$-value \\
\hline \multicolumn{4}{|c|}{ Perpendicular height } \\
\hline$<3 \mathrm{~mm}$ & 11 & 3 & .008 \\
\hline 3-5 mm & 41 & 9 & \\
\hline $6-8 \mathrm{~mm}$ & 46 & 12 & \\
\hline 9-11 mm & 24 & 7 & \\
\hline $12-14 \mathrm{~mm}$ & 22 & 8 & \\
\hline$\geq 15 \mathrm{~mm}$ & 21 & 11 & \\
\hline Daughter sac & 7 & 5 & .10 \\
\hline Multiple lobes & 11 & 3 & .87 \\
\hline \multicolumn{4}{|l|}{ Aneurysm angle } \\
\hline$<46$ & 54 & 15 & .79 \\
\hline $46-90$ & 28 & 7 & \\
\hline $91-135$ & 41 & 14 & \\
\hline $136-180$ & 73 & 18 & \\
\hline \multicolumn{4}{|l|}{ Neck diameter } \\
\hline$<3 \mathrm{~mm}$ & 18 & 3 & .45 \\
\hline 3-5 mm & 100 & 31 & \\
\hline $6-8 \mathrm{~mm}$ & 34 & 12 & \\
\hline 9-11 mm & 10 & 3 & \\
\hline$>11 \mathrm{~mm}$ & 6 & 1 & \\
\hline \multicolumn{4}{|l|}{ Aspect ratio } \\
\hline$<1$ & 7 & 2 & .28 \\
\hline 1 & 70 & 18 & \\
\hline 2 & 46 & 12 & \\
\hline 3 & 27 & 12 & \\
\hline $4-5$ & 11 & 4 & \\
\hline $6-8$ & 3 & 1 & \\
\hline \multicolumn{4}{|l|}{ Size ratio } \\
\hline$<1$ & 7 & 1 & .01 \\
\hline 1 & 23 & 6 & \\
\hline 2 & 22 & 7 & \\
\hline 3 & 32 & 9 & \\
\hline $4-5$ & 59 & 13 & \\
\hline $6-8$ & 41 & 7 & \\
\hline$>9$ & 14 & 14 & \\
\hline \multicolumn{3}{|c|}{ Direction of max diameter, anterior-posterior } & .33 \\
\hline \multicolumn{3}{|c|}{ Direction of max diameter, cephalad-caudad } & .61 \\
\hline \multicolumn{3}{|c|}{ Direction of max diameter, medial-lateral } & .54 \\
\hline \multicolumn{3}{|c|}{ Parent vessel diameter } & .06 \\
\hline \multicolumn{3}{|c|}{ Calculated aneurysm volume } & .41 \\
\hline
\end{tabular}

both UIA size and location cannot be determined, given the current matching for those factors; an analysis of the complete ISUIA dataset will be performed to clarify this issue. The present study does suggest that other morphological characteristics should be considered besides just maximal diameter and location when counseling patients regarding the rupture risk of UIA.

An additional important finding in this study is the role of SR in rupture risk stratification. The SR calculation was found to be highly associated with rupture in univariate analyses, but did not achieve significance on multivariate regression analysis. This phenomenon is not unexpected, as the analysis (intentionally) partially controlled for both the denominator and the numerator in the SR calculation by matching aneurysms by size and parent vessel location. Despite this dilution due to matching, SR was a significant predictor on univariate analysis, suggesting a highly significant association between SR and rupture. Furthermore, by controlling for aneurysm parent vessel location and therefore partially controlling for the denominator in the SR calculation, the fact that perpendicular height was significant on multivariate regression underscores the importance of $S R$ in rupture risk, given that perpendicular height often approximates the numerator in the SR calculation. The results of this analysis are consistent with recent prospective and retrospective studies suggestion that $\mathrm{SR}$ is a predictor of UIA rupture. ${ }^{7-9}$

The increased rupture risk with increasing SR, as demonstrated in both this study and others, is thought to be secondary to more dangerous hemodynamic characteristics present in aneurysms with higher SR. ${ }^{10}$ In one descriptive flow study using computational fluid dynamics modeling, SR was a significant predictor of aneurysm rupture apart from wall shear stress or the oscillatory shear index. ${ }^{11}$ Considering the results published by Carter and colleagues $^{6}$ showing differing ruptured aneurysm sizes based on location, it is plausible that SR may potentially account for these discrepancies. For instance, an aneurysm measuring $4 \mathrm{~mm}$ on the internal carotid artery, where the vessel diameter is $4 \mathrm{~mm}$ (SR of 1), may have a lower risk of rupture than a 4-mm aneurysm on the anterior cerebral artery, where the parent vessel measures only $1.5 \mathrm{~mm}$ (SR of 2.7). One potential benefit of the SR calculation over other more complicated computerized models is the ease with which it can be reliably calculated by 2 -dimensional angiography. 8,12

\section{Limitations}

This study has important limitations. First, the matching of cases and controls was limited to the patients within the ISUIA cohort and is not exact (see Table 1). Second, despite the large cohort size, there were only 57 aneurysms with rupture, limiting the morphological analysis. Third, measurements were obtained from 2-dimensional angiograms, which increases measurement error for morphological factors compared to 3-dimensional angiography. When controlling for factors such as size and then assessing for the impact for other factors such as SR and perpendicular height, the statistical analysis becomes complicated (colinear) and somewhat confounded. Our statistical findings should be interpreted with this in mind. Finally, family history, age, and smoking history were not evaluated in the analysis.

\section{Generalizability}

As this study is only a sample of the ISUIA cohort, the study has limited generalizability. Reanalysis of the entire ISUIA database for SR and other factors will be necessary to provide adequate external validity. 


\section{CONCLUSION}

In a case-control study of 57 ruptured and 198 UIA matched based on size and location from the ISUIA cohort, SR and perpendicular height were identified as significant predictors of aneurysm rupture on univariate analysis. On multivariate analysis, perpendicular height was the only significant predictor of rupture. This study underscores the importance of other morphological factors in aneurysm rupture besides greatest diameter and anterior vs posterior location. Future analysis of the ISUIA database focusing on these additional factors will be helpful in providing further insight into the rupture risk of UIA.

\section{Disclosures}

This work is supported by grants (NS068092 "Predictors of Long-Term Outcome of Unruptured Intracranial Aneurysms," and NS028492 "Unruptured Intracranial Aneurysms: Neurologic Outcome") from the National Institute of Neurological Disorders and Stroke. Dr Mocco serves as a consultant to Cerebrotech, Pulsar, TSP Inc, and Rebound Medical. Dr Mocco has investor interests in Blockade Medical and TSP Inc. The authors have no personal, financial, or institutional interest in any of the drugs, materials, or devices described in this article.

\section{REFERENCES}

1. International study of unruptured intracranial aneurysms investigators. Unruptured intracranial aneurysms-risk of rupture and risks of surgical intervention. $N$ Engl J Med. 1998;339:1725-1733.

2. Wiebers DO, Whisnant JP, Huston J, 3rd, et al. Unruptured intracranial aneurysms: natural history, clinical outcome, and risks of surgical and endovascular treatment. Lancet. 2003;362:103-110.

3. Juvela S, Porras M, Poussa K. Natural history of unruptured intracranial aneurysms: probability of and risk factors for aneurysm rupture. $J$ Neurosurg. 2000;93:379-387.

4. Investigators UJ, Morita A, Kirino $\mathrm{T}$, et al. The natural course of unruptured cerebral aneurysms in a japanese cohort. N Engl J Med. 2012;366:2474-2482.

5. Weir B, Disney L, Karrison T. Sizes of ruptured and unruptured aneurysms in relation to their sites and the ages of patients. J Neurosurgery. 2002;96:64-70.

6. Carter BS, Sheth S, Chang E, Sethl M, Ogilvy CS. Epidemiology of the size distribution of intracranial bifurcation aneurysms: smaller size of distal aneurysms and increasing size of unruptured aneurysms with age. Neurosurgery. 2006;58:217223; discussion 217-223

7. Kashiwazaki D, Kuroda S, Sapporo SAHSG. Size ratio can highly predict rupture risk in intracranial small $(<5 \mathrm{~mm})$ aneurysms. Stroke. 2013;44:2169-2173.

8. Rahman M, Smietana J, Hauck E, et al. Size ratio correlates with intracranial aneurysm rupture status: a prospective study. Stroke. 2010;41:916-920.

9. Tykocki T, Nauman P, Dow Enko A. Morphometric predictors of posterior circulation aneurysms risk rupture. Neurol Res. 2014;36:733-738.

10. Tremmel M, Dhar S, Levy EI, Mocco J, Meng H. Influence of intracranial aneurysm-to-parent vessel size ratio on hemodynamics and implication for rupture: results from a virtual experimental study. Neurosurgery. 2009;64:622-630; discussion 630-621.

11. Xiang J, Natarajan SK, Tremmel M, et al. Hemodynamic-morphologic discriminants for intracranial aneurysm rupture. Stroke. 2011;42:144-152.

12. Prestigiacomo CJ, He W, Catrambone J, et al. Predicting aneurysm rupture probabilities through the application of a computed tomography angiographyderived binary logistic regression model. J Neurosurg. 2009;110:1-6.

\section{COMMENT}

This is an important study that uses a nested case control study ISUIA data in an attempt to determine factors other than aneurysm size and location that may help predict aneurysm rupture. The authors' results confirm the importance of perpendicular height and size ratio as risk factors for rupture. They clearly note the limitations of their study and accurately portray their work as 1 step in a thorough analysis of aneurysm features that may predispose to rupture.

Their study, like other studies of unruptured intracranial aneurysms (UIAs), is limited by the selection bias that exists. ${ }^{1,2}$ Expert physicians and surgeons recommend invasive treatment for patients with UIAs if they think the risk of aneurysm rupture outweighs the risk of treatment. Therefore, if the treating physicians are correct, those aneurysms selected for observation should have a very low risk of rupture and it is impossible to state what the rupture risk for the treated aneurysms would have been if they had been selected for observation. We have experienced the same phenomenon in our studies of patients with UIAs, for whom observation rather than invasive treatment was recommended. ${ }^{2}$

A randomized study of all patients with UIAs would help answer questions about best treatment but such a study is impractical and would likely give us unreliable information. Clinicians evaluating patients with UIAs do not have equipoise in regard to treatment versus observation, and those patients thought to be at higher risk of rupture would be treated outside the trial. If only patients thought to have a low risk of rupture were randomized the trial would reliably demonstrate that observation was preferable to treatment. As this study would be classified as "Level I evidence", we would then no longer be able to offer invasive treatment to those patients who might actually benefit.

In the ideal situation, randomized controlled trials (RCTs) let us make causal inferences in a way that no other study design can. However, surgical RCTs have real problems in regard to intention to treat versus as treated analyses, lack of masking, lack of equipoise in those recommending treatment, the variability of surgical skill and judgement, the intermittent nature of RCTs when invasive and non-invasive therapies are changing, the duration of the study for prophylactic surgical procedures and how all of these factors affect the generalizability of the study results.

I believe there is a better option than the RCT for certain problems such as management of UIAs. One can design a registry in such a way that reliable causal inferences can be made using propensity score matching. ${ }^{1}$ The questions we need to address to create a registry that would allow us to make causal inferences about treatment options for unruptured aneurysms would include the following:

1. What randomized study do we want to model? Invasive treatment vs observation of UIAs would be the model.

2. Who are the decision makers for treatment assignment? Patients, patients' families, and physicians are decision makers.

3. What are the key covariates that are used for treatment assignment? Numerous aneurysm-specific, patient-specific, and physicianspecific variables need to be assessed.

4. Can we quantify the co-variates? Yes.

5. What clinically meaningful outcomes do we want to measure? Functional health status, mortality, and aneurysm rupture would be reasonable.

6. What sample size will be necessary? Our preliminary power analysis indicates a minimum number of 870 observed, 435 clipped and 435 coiled patients.

7. How long would the registry need to run? A minimum of 5 years would be needed.

8. What established registry infrastructure is available? The NeuroPoint Alliance has the needed registry infrastructure. 
If we could create such a registry I believe we would be able to make more reliable causal inferences regarding the best treatment options for patients with UIAs.

Robert E. Harbaugh State College, Pennsylvania
1. Harbaugh RE. Rupture risk of unruptured aneurysms: beyond size and shape. J Neurosurg. 124: 286-287, 2016.

2. Ramachandran M, Retarekar R, Berkowitz B, et al. Assessment of image-derived risk factors for natural course of unruptured cerebral aneurysms. J Neurosurg. 124: 288-295, 2016. 